\title{
The dependence of wear rate on wear scar size in fretting; the role of debris (third body) expulsion from the contact
}

\author{
T. Zhu, P.H. Shipway*, W. Sun \\ Faculty of Engineering, University of Nottingham, UK
}

\begin{abstract}
The paper 'The third-body approach: a mechanical view of wear' by Maurice Godet (Wear, 100 (1984), pp 437-452) was perhaps the first to articulate clearly the key role of the rate of debris expulsion from a fretting contact in controlling the overall rate of wear. Whilst subsequent research over the past four decades has acknowledged this, the issue is generally addressed qualitatively rather than quantitatively. There are many parameters which will affect the rate of debris expulsion from a fretting contact, and amongst them is the physical size of the fretting contact. In this paper, for the first time, a physically-based relationship is proposed between the debris-expulsion limited wear rate and the contact size. This relationship is able to account for differences in wear rates observed in tests conducted with different (and evolving) contact geometries (non-conforming contacts) over a range of durations, thus clearly demonstrating the validity of the approach.
\end{abstract}

Keywords: Fretting; debris; modelling, debris flow

*Corresponding author: philip.shipway@nottingham.ac.uk

\section{Introduction}

Fretting is defined as the small-amplitude oscillatory motion between components in contact with each other [1]. Fatigue and wear are the normal damage modes associated with fretting, being commonly encountered where system vibrations are present across a range of industry sectors, such as aerospace and energy generation [2]. Fretting wear can cause loss of material, which in turn impairs the functionality of a system. Hence, a thorough understanding of fretting wear is necessary in order to facilitate the development of a predictive wear model.

As outlined by Meng and Ludema [3], hundreds of wear models have been proposed during the last five decades. Unfortunately, very few of them can be considered as robust enough to predict wear. In terms of fretting wear specifically, there are two main approaches that have been widely accepted: (i) the Archard wear model [4] along with the energy-wear formulation derived from it $[5,6]$, and (ii) the third-body approach introduced by Godet [7]. Classically, wear is considered to be a process of particle detachment from the surfaces in contact. This is the central concept of Archard's wear model (initially derived for sliding wear, and not for fretting wear) [4], which associates the amount of material removal from the surface, i.e. the 'wear volume', with the sliding distance and the applied load. As noted by Fillot et al. [8], concepts and terms used in the fretting literature, such as 'wear rate' and 'wear mechanism', were inherited from the classical Archard approach, where the 'wear rate' describes the rate of material removal from the surfaces. However, in fretting, this debris is easily trapped in the contact region due to the small displacement amplitude (typically tens of micrometres) [9], with the removal of this debris being required for further wear to take place. Therefore, it is argued that the Archard approach (without the consideration of the removal of debris from the contact) is not able to account for the differences in wear rates associated with differences in debris entrapment in the contact. 
The concept of the third-body in fretting was introduced by Godet in 1984 [7], who argued that the wear process and rate are the result of the competition between debris formation and ejection. In his early work, Hurricks [9] proposed that the wear particles are first detached from the surface and then oxidised and ground into fine debris, finally forming a debris layer. This layer was referred to as the 'third body' by Godet [7], who suggested that the presence of the third body changes wear behaviour completely: it acts as a solid lubricant to separate the contacting bodies (i.e. the first bodies), carries load, accommodates velocity differences between the first bodies, and limits (or even prevents) further degradation of the surfaces of the first bodies. Many parameters can influence the formation and ejection of the third body debris, which consequently alters the wear behaviour. As Godet noted later [10], the classical definition of 'wear' as the detachment of particles is only part of the wear process. Indeed, a complete description of the wear process should include debris formation, role of debris in modifying the contact and debris ejection from the contact. In general, the commonly used term 'wear rate', simply refers to the ratio linking the measured wear volume with the tribological system parameters [1]. Since fretting wear itself represents the consequences of both debris formation and ejection, it should be noted that the measured wear volume contains the information related to the complete wear process. Berthier and co-workers $[8,11]$ therefore suggested that it is not appropriate to interpret 'wear rate' as the rate of particles detachment, illustrating this concept via a tribology circuit as shown in Figure 1(a). Here, the wear process is considered as a pipeline, starting with particle detachment from first bodies and continuing with the formation of the third body; finally, depending upon the accumulation in the contact and other parameters that may influence the contact condition, the third body is ejected from the contact at a certain rate. It is, therefore, argued that the 'wear rate' is controlled by the balance between the corresponding debris formation ejection rates. Figure $1(b)$ indicates that the system will tend towards an equilibrium between debris formation and debris ejection [8]; any change in contact conditions would trigger an increase or decrease in the corresponding rate of debris formation or ejection, altering the equilibrium state and influencing the observed 'wear rate'.

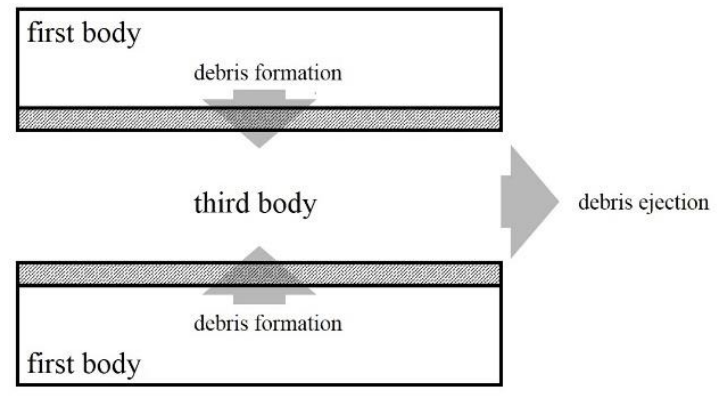

(a)

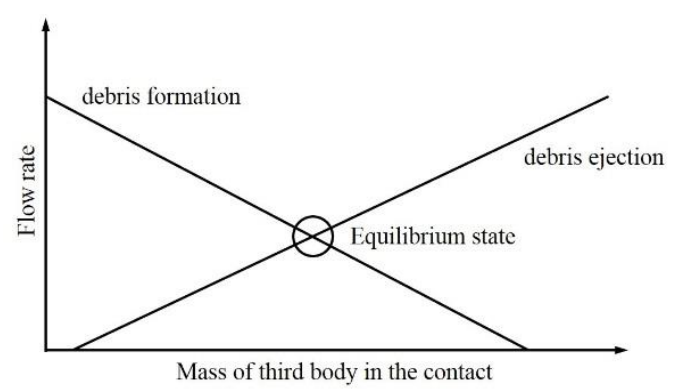

(b)

Figure 1 (a): Berthier's illustration of the tribology circuit [11]; (b): illustration of the competition between debris formation and debris ejection [8] for a fretting contact.

Waterhouse suggested that fretting contacts can be divided into two categories depending on the behaviour of oxide debris [12]. A surface with shallow dish-like depressions is formed if debris are able to escape from the contact, whilst entrapment of debris leads to small but deep holes. More recent work [13-16] has examined the effects of various tribological system parameters in fretting, such as displacement amplitude, temperature and oscillation frequency. Irrespective of their focus, the conclusions of these studies converge on a discussion of how the tribological system parameters influence the formation and ejection of debris, and how a change in debris retention or ejection can alter the wear behaviour and the 'wear rate'. However, relatively little work has investigated the effect of contact geometry, despite the fact that a large variety of contact geometries have been employed in experimental research. In general, these contact geometries can be divided into two primary categories, namely non-conforming contacts and conforming contacts. The use of non-conforming contacts (e.g. ball-on-flat and cylinder-on-flat) is very common because it replicates contact types seen 
in many service conditions and also aids reproducible laboratory experimentation in that it reduces problems associated with specimen alignment. As observed by Fouvry and co-workers $[5,17]$ and Warmuth et al. $[13,14]$, the observed 'wear rate' was significantly reduced with increasing contact conformity (i.e. as the radius of curvature of the non-plane body increased). They argued that such reduction in wear rate is associated with the increased entrapment of wear debris that occurs as contact conformity is increased, and thus focussed on the role of the third body in line with the work of Godet [7] and Berthier et al. [8, 11, 18]. The third-body approach, together with more recent work on the effect of contact geometry in fretting, point to the key roles of debris kinematics and contact geometry in the fretting wear process.

It is recognised that when a non-conforming geometry is employed in fretting research, the contact size increases continuously as wear occurs, which in turn, is expected to influence the balance between debris formation and ejection. It is suggested that the observed wear rate is the lesser of (i) the rate of removal from the wearing surfaces to form debris and (ii) the rate of removal of that debris from the contact; a schematic diagram illustrating this hypothesis is presented in Figure 2. In cases where debris ejection is the rate-determining step, the contact size will thus exert an influence on the 'wear rate'. In such cases, the observed 'wear rate' is thus a function of the amount of wear (as opposed to being assumed to be constant).

In this paper, a cylinder-on-flat contact geometry was employed, and the evolving contact was characterised by the wear scar width. A wide range of the number of cycles (from $5 \times 10^{3}$ cycles to $5 \times$ $10^{6}$ cycles) and two different initial geometries (cylinders with $6 \mathrm{~mm}$ and $160 \mathrm{~mm}$ radii) were employed to obtain different wear scar widths and rates of scar width development. It is argued that changing wear scar width should alter the rate of debris ejection from the contact, and thus control the observed 'wear rate' when debris ejection is rate-determining. An analytical wear model is presented which describes the dependence of the wear rate on wear scar width for a cylinder-on-flat contact across a range of wear scar widths, which are associated with both different initial geometries and different amounts of wear.

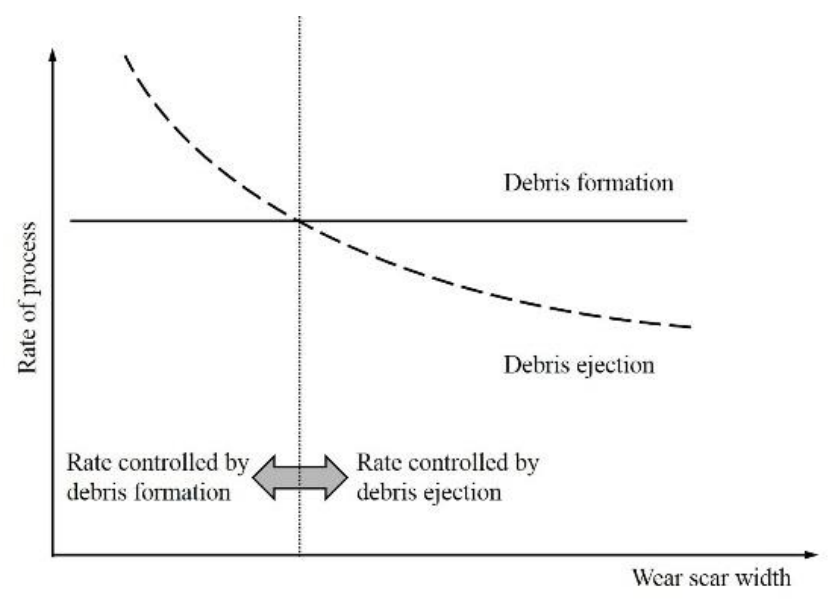

Figure 2: Schematic diagram illustrating the dependence of rates of wear and debris ejection on wear scar width, with regions where debris formation and debris ejection are the rate-determining processes (i.e. the process with the lower of the two rates at any scar width).

\section{Experimental procedure}

\subsection{Materials and test procedures}

All of the specimens used in this work were produced from a high strength alloy steel (BS S132). The chemical composition and mechanical properties of $S 132$ are presented in Tables 1 and 2 [19, 20]. 
Table 1: Chemical composition of the high strength steel (wt.\%) [19].

\begin{tabular}{|c|c|c|c|c|}
\hline$C$ & Mo & V & $\mathrm{Cr}$ & $\mathrm{Si}$ \\
\hline $0.35-0.43$ & $0.8-1.10$ & $0.15-0.25$ & $3.0-3.5$ & $0.1-0.35$ \\
\hline $\mathrm{Mn}$ & $\mathrm{Ni}$ & $\mathrm{P}$ & $\mathrm{S}$ & $\mathrm{Fe}$ \\
\hline $0.4-0.7$ & $<0.3$ & $<0.007$ & $<0.002$ & Balance \\
\hline \multicolumn{5}{|c|}{ Table 2: Mechanical properties of the high strength steel [20]. } \\
\hline$\sigma_{y} / \mathrm{MPa}$ & $\sigma_{u t} / \mathrm{MPa}$ & HV30 & $E / \mathrm{GPa}$ & $v$ \\
\hline 1247 & 1697 & $485 \pm 10$ & 206.8 & 0.28 \\
\hline
\end{tabular}

The steel was subjected to a commercially relevant heat treatment cycle, the details of which can be found in a previous paper [19]. The specimens were subsequently ground into flat and cylindrical specimens as shown in Figure 3. All of the specimens had a width of $10 \mathrm{~mm}$, while the cylindrical specimens were manufactured with radii of both $6 \mathrm{~mm}$ and $160 \mathrm{~mm}$. The Vickers hardness (HV30) of the surface was measured to ensure that the required surface hardness (see Table 2) had been achieved.

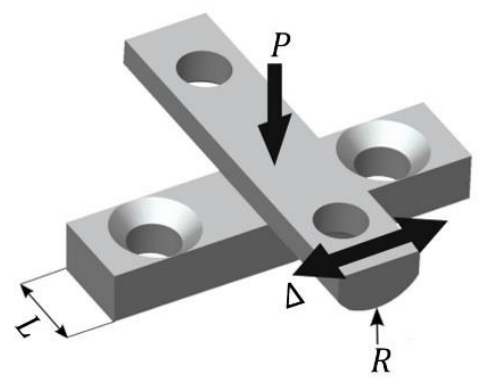

Figure 3: Illustration of the cylinder-on-flat specimen configuration applied in fretting tests: $L$ is the length of the line contact, $10 \mathrm{~mm} ; \boldsymbol{R}$ is the radius of the cylindrical specimens, namely $6 \mathrm{~mm}$ or $160 \mathrm{~mm} ; \boldsymbol{P}$ is the normal load, $450 \mathrm{~N}$; $\Delta^{*}$ is the applied displacement.

The preparation work on specimens involved thorough demagnetisation and surface degreasing, before being rinsed with industrial methylated spirit (IMS) and dried. Specimens were arranged in a cylinder-on-flat configuration for the fretting tests (Figure 3) producing an initial line contact with a length of $10 \mathrm{~mm}$. The flat specimen was mounted on a stationary lower specimen mounting block (LSMB) and the cylindrical specimen was attached to a moving upper specimen mounting block (USMB). A constant normal load, $P$, was applied to the USMB through a dead weight. An oscillatory motion with an applied displacement, $\Delta$ (with $\Delta^{*}$ referring to the applied displacement amplitude), was generated by an electromagnetic vibrator (EMV) and applied to the USMB. Throughout the test, the relative displacement $(\Delta)$ between the USMB and LSMB was monitored using a capacitance displacement sensor; the tangential traction force across the specimen pair contact, $Q$ (with amplitude $Q^{*}$ ), was measured by a piezoelectric load cell. Both the tangential traction force and the relative displacement were sampled at a rate of 200 measurements for each fretting cycle. These data were used to plot fretting loops, derive the actual slip amplitude $\left(\delta^{*}\right)$ and calculate the energy dissipated in the contact $\left(E_{d}\right)$ for each cycle based on methods proposed by Fouvry et al. [5, 6]. Fretting tests were carried out with both $6 \mathrm{~mm}$ and $160 \mathrm{~mm}$ radius specimens against a flat specimen (termed R- 6 pairs and R-160 pairs, respectively). All tests were conducted with the same normal load, applied displacement amplitude, and frequency over a range of test durations from $5 \times 10^{3}$ cycles to $5 \times 10^{6}$ cycles at ambient temperature. Test conditions are summarised in Table 3.

Table 3: Summary of the fretting test conditions.

$\begin{array}{ll}\text { Normal load }(P) / \mathrm{N} & 450 \\ \text { Displacement amplitude }\left(\Delta^{*}\right) / \mu \mathrm{m} & 50 \\ \text { Cylindrical specimen radius }(R) / \mathrm{mm} & 6,160\end{array}$




\begin{tabular}{ll}
\hline Test duration $(N) / \times 10^{3}$ cycles & $5,20,100,500,1000,2000,5000$ \\
Frequency $(f) / \mathrm{Hz}$ & 20 \\
\hline
\end{tabular}

\subsection{Characterization of wear scars and debris}

Following a fretting test, the wear volume and the wear scar surface topography of specimens were evaluated via profilometry, whilst the nature of the wear scar and the distribution of wear debris were characterised using scanning electron microscopy (SEM). Specimens were rinsed with IMS to remove loose wear debris before examination. A Bruker Contour GT-I interferometer, with a vertical resolution of $0.15 \mathrm{~nm}$ and a lateral resolution of $4 \mu \mathrm{m}$ was used to profile the wear scar. The scanned areas were $11 \mathrm{~mm}$ and $10 \mathrm{~mm}$ in width for the scars on R-6 cylinders and R-160 cylinders, respectively. In order to estimate the wear volume, a reference surface must be determined to represent the surface before the fretting process. For both flat and cylindrical specimens, the reference surfaces were defined as the best-fit plane of the surface profiles outside of the wear scar. As suggested by Elleuch and Fouvry [21], the material build-up above the reference surface is regarded as transferred material and is regarded as the positive volume $\left(V^{+}\right)$; in contrast, the loss of material below the reference plane is regarded as the negative volume $\left(V^{-}\right)$. The definitions of material lost and material transferred are illustrated in Figure 4 a. The calculation of the overall net wear volume $\left(V^{W}\right)$ is as defined in Equation 1 , where the subscripts refer to the wear and transfer volumes on the respective specimens. Average profiles across scars were generated by taking the average of individual profiles across the full scar width. As illustrated in Figure 4a (schematically) and Figure 4b (using example data from an R-160 pair following $5 \times 10^{6}$ fretting cycles), the wear scar width was determined from the average profile of the wear scar on the flat specimen and defined as the width of the central portion of the wear scar that lay below the reference surface.

$$
\begin{gathered}
V^{+}=V_{\text {flat }}{ }^{+}+V_{\text {cylindrical }}{ }^{+} \\
V^{-}=V_{\text {flat }}{ }^{-}+V_{\text {cylindrical }}{ }^{-} \\
V^{W}=-\left(V^{+}+V^{-}\right)
\end{gathered}
$$

SEM was used to characterise the wear scar and the distribution of the oxide debris using a Philips XL30 microscope. Images were taken using both secondary electron (SE) and backscattered electron (BSE) imaging techniques. BSE imaging is particularly useful since it allows oxide debris (with its lower average atomic number) to be readily distinguished from the metallic substrate as a result of its lower imaging brightness. 


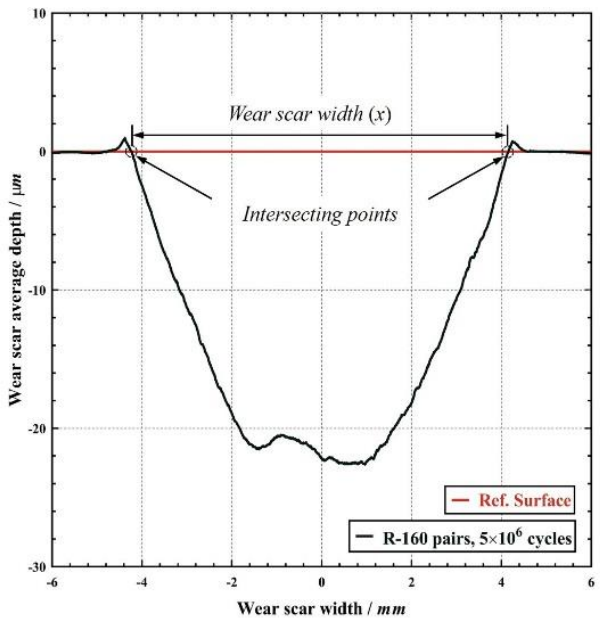

(a)

(b)

Figure 4: (a) Illustration of the assessment of material lost and material transferred with respect to the reference surface and the definition of wear scar width; (b) example of the determination of wear scar width from the average surface profile for the R-160 pairs test over $5 \times 10^{6}$ cycles.

\section{Experimental results}

Figure 5 shows $V^{W}$ as a function of $E_{d}$ for both the R- 6 pairs and the R-160 pairs. It can be seen that, for equivalent dissipated energies, wear volumes are higher for the R-6 pairs than for the R-160 pairs. Moreover, for both pair types, it can be seen that the wear rate (wear volume per unit energy dissipated) decreases with increasing energy dissipated. Figure $5 \mathrm{~b}$ illustrates that whilst the wear volume increases with dissipated energy from the outset for R-6 pairs, there is an incubation period for the R-160 pairs of at least $\sim 5 \mathrm{~kJ}$ before a measurable wear volume was observed. It should be noted that several (up to six) replicated tests were carried out to investigate repeatability. It was found that the repeatability (as defined by the standard error in the mean) of both dissipated energy and net wear volume was always less than $10 \%$, and in some cases much less.

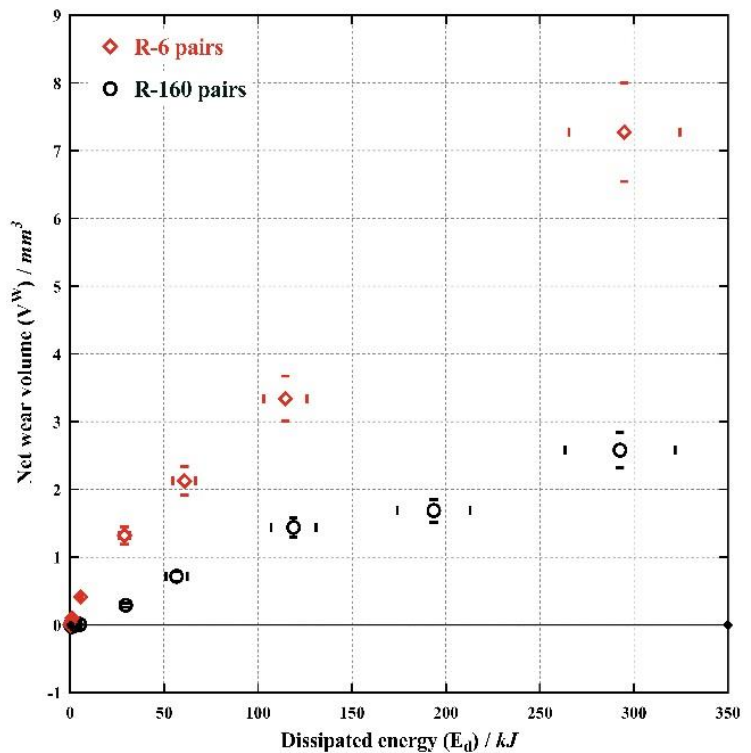

(a)

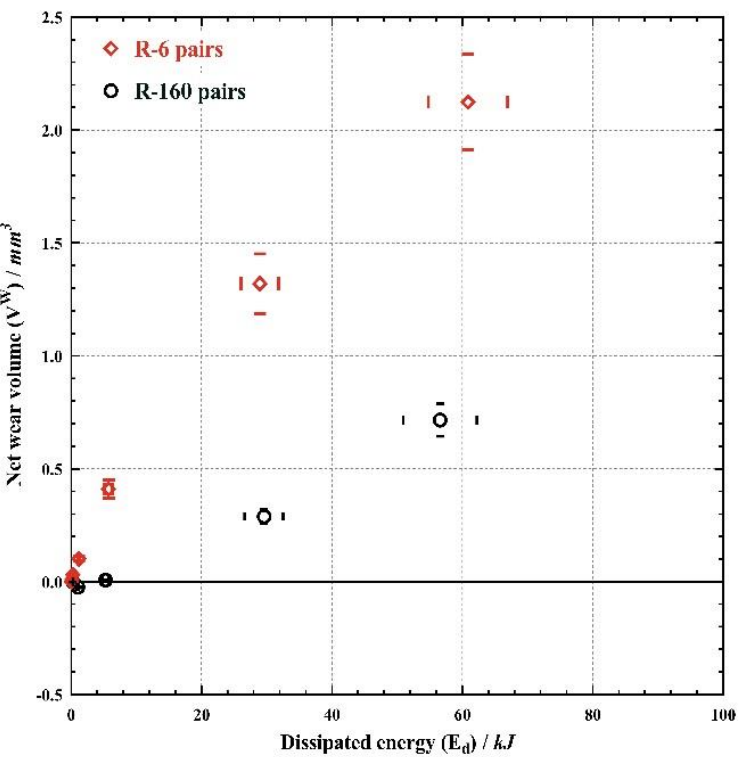

(b) 
Figure 5: Net wear volume as a function of dissipated energy for fretting tests conducted with R-6 pairs and R160 pairs with the range of repeatability indicated for data points: (a) data from all tests conducted in this work; (b) detailed view for tests where the dissipated energy was less than $100 \mathrm{~kJ}$, giving a clearer view of the initiation behaviour.

Figure 6 presents the 3-D profilometric representations of the wear scars on the flat specimens for selected tests with both R-6 pairs and R-160 pairs as a function of test duration. Each scanned area is $11 \mathrm{~mm} \times 10 \mathrm{~mm}$. The magnification normal to the plane is 40 times greater than that within the plane. For the R- 6 pairs, the wear scars are seen to be of uniform width for all examined test durations, and they grow in both width and depth as the number of cycles increases. However, as observerd previously [14], the more-conforming R-160 pairs exhibit the formation of localised pit-peak features in the early stages of the wear scar development (from $5 \times 10^{3}$ to $1 \times 10^{5}$ cycles); these features result from a metallic transfer between the specimens. It is notable that these features have largely been eliminated after $1 \times 10^{6}$ cycles, following which a wide, but relatively uniform wear scar is observed. 

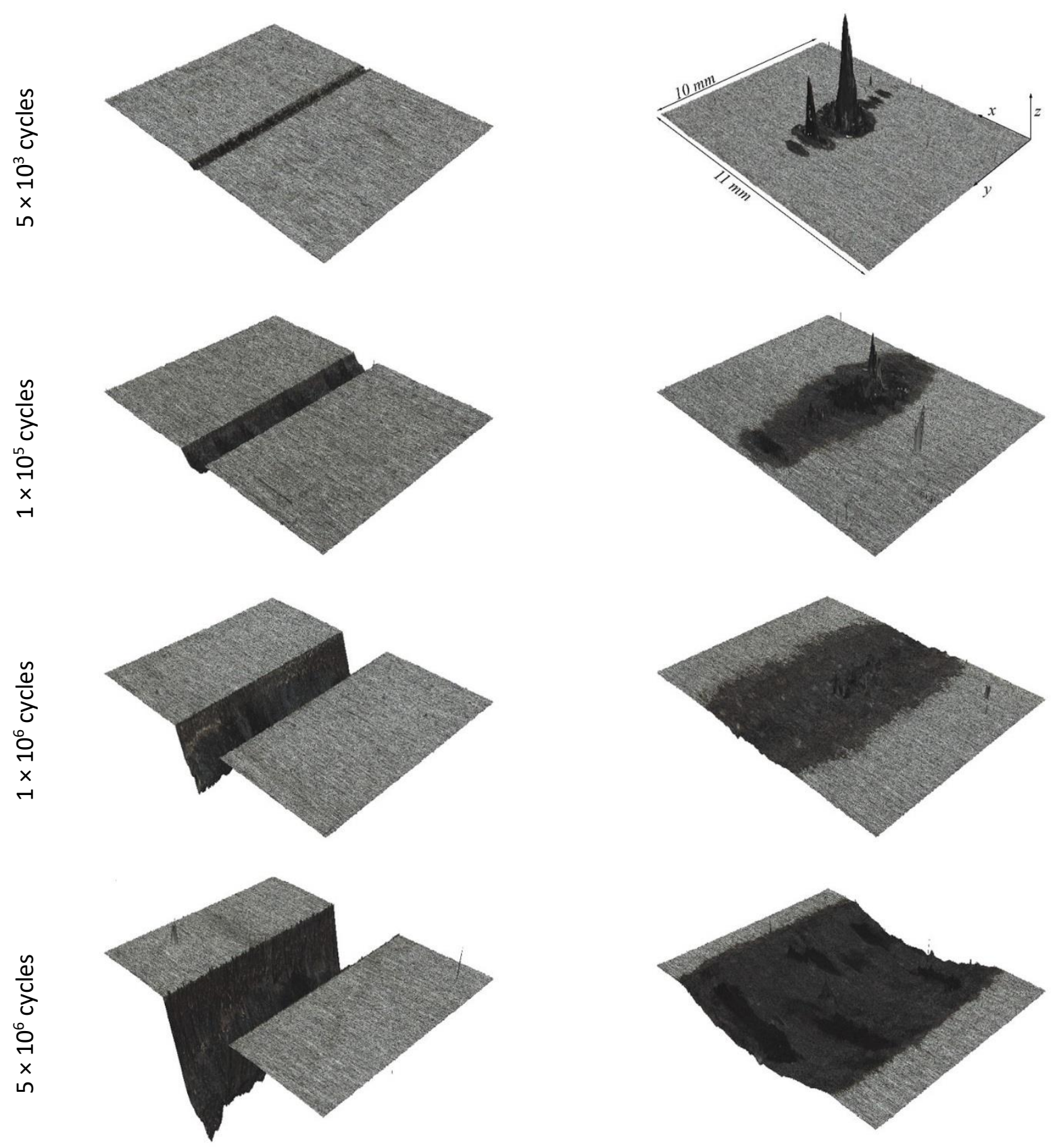

(a) R-6 pairs

(b) R-160 pairs

Figure 6: Surface topography images of wear scar on flat specimens for selected fretting tests conducted over a range of test durations, from $5 \times 10^{3}$ cycles to $5 \times 10^{6}$ cycles: (a) R-6 pairs; (b) R-160 pairs. The magnification in all images is the same in the z-direction (to provide scale, the height of the peak in the R-160 pair after $5 \times$ $10^{3}$ cycles is $\sim 150 \mu \mathrm{m}$ ). 


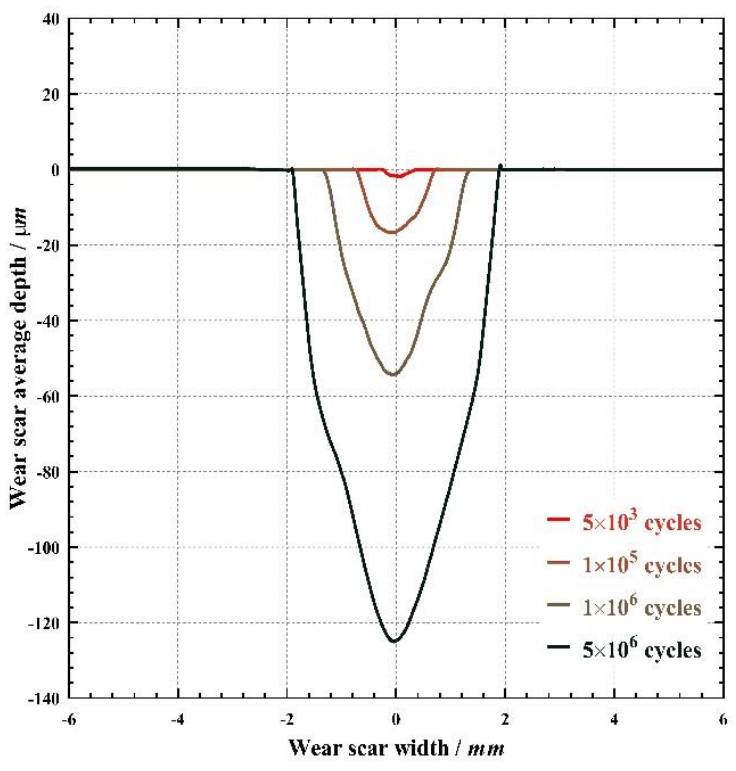

(a)

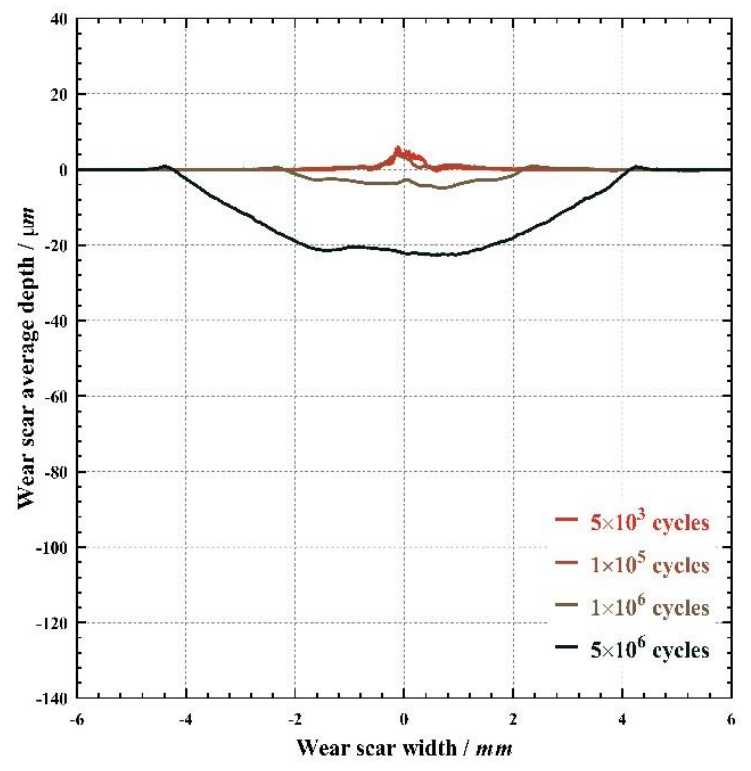

(b)

Figure 7: Averaged profiles of fretting wear scar on flat specimens for selected fretting tests conducted over a range of test durations, from $5 \times 10^{3}$ cycles to $5 \times 10^{6}$ cycles; (a) R-6 pairs; (b) R-160 pairs.

Figure 7 shows the average profiles of wear scars on flat specimens (derived from the data of the type presented in Figure 6) for fretting tests conducted with R-6 and R-160 pairs. Figure 7a illustrates that a U-shaped wear profile for R- 6 pairs is formed as early as after $5 \times 10^{3}$ fretting cycles, and this then develops in depth and width as the number of cycles increases. In contrast, for R-160 pairs, tests with up to $1 \times 10^{5}$ cycles show no significant material removal from the flat specimen; the development of a U-shaped wear scar is clearly observed after $1 \times 10^{6}$ cycles, growing in both depth and width as the cycle count increases.

Figure 8 shows BSE-SEM images of the wear scars on the flat specimens for the R- 6 (left column) and R-160 (right column) specimen pairs as a function of test duration. In this imaging mode, oxide debris has a lower contrast than metallic debris in the fretting scars due to its lower average atomic number. For the R-6 pairs, it can be seen that the fretting scar is only sparsely covered in oxide after $5 \times 10^{3}$ cycles, but that an oxide debris bed has fully covered the scar following $1 \times 10^{5}$ cycles. This oxide bed is seen to be non-uniform, with evidence at $5 \times 10^{6}$ cycles that the oxide delaminates locally and thus exposes the underlying metallic material to further wear. In contrast, in the R-160 pairs, the pit-peak features observed in the early stages $\left(5 \times 10^{3}\right.$ cycles) are seen to have a largely metallic character, indicating that they are formed by metallic transfer between the specimens. After $1 \times 10^{5}$ cycles, the surface is largely covered by an oxide debris bed, although patches where there is no oxide coverage exist. This situation remains after $1 \times 10^{6}$ cycles, and it is only after $5 \times 10^{6}$ cycles that the oxide coverage in the fretting scar is largely uniform. Indeed, after $5 \times 10^{6}$ cycles, there are no significant differences in the oxide coverage in the wear scar between the R- 6 and the R-160 pairs. 

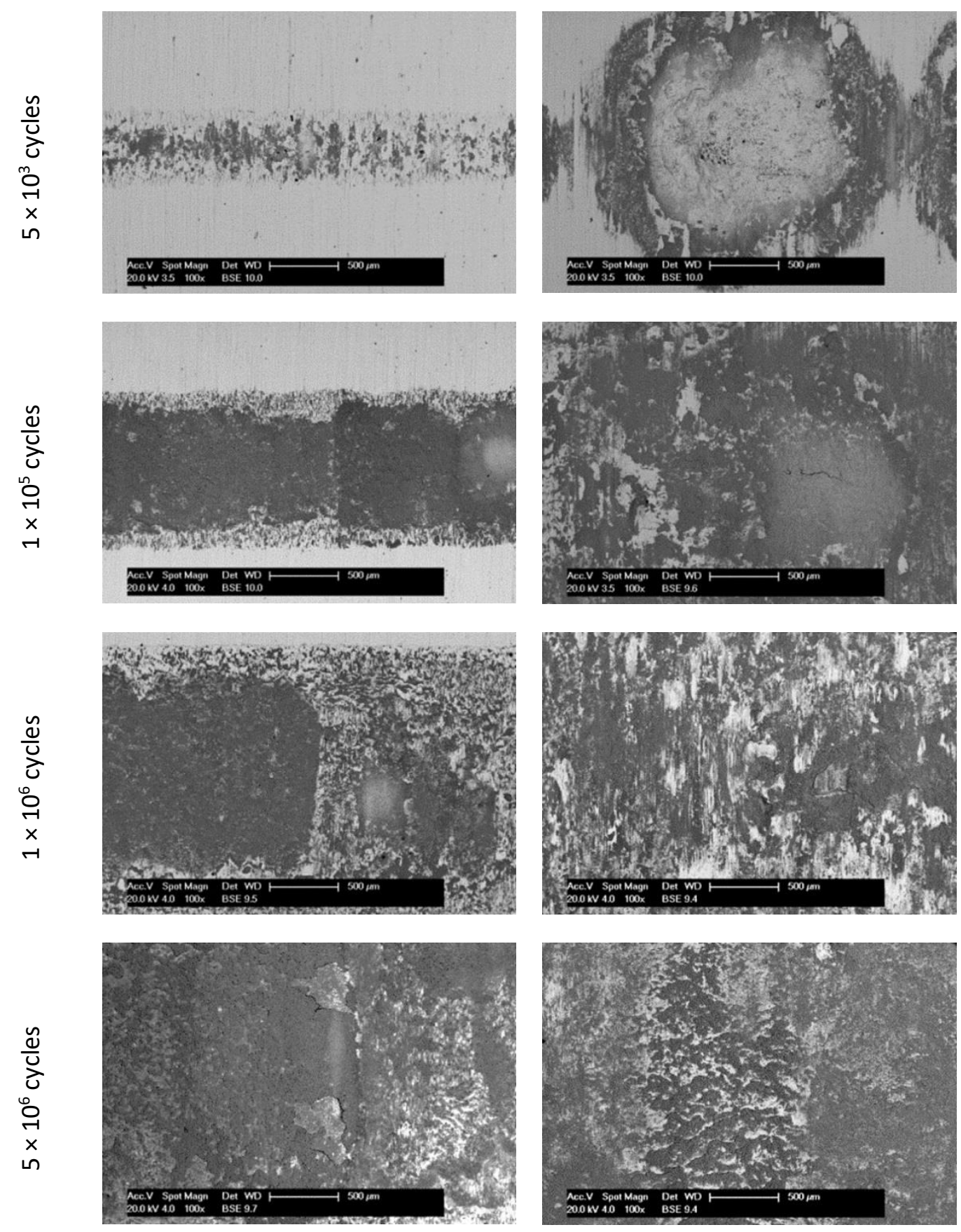

(a) R-6 pairs

(b) R-160 pairs

Figure 8: BSE images of the top view of the fretting wear scar on flat specimens for fretting tests conducted over a range of test durations, from $5 \times 10^{3}$ cycles to $5 \times 10^{6}$ cycles with (a) R-6 specimen pairs; (b) R-160 specimen pairs.

\section{Development of the model}

\subsection{Link between wear scar width and wear volume in a cylinder-on-flat contact}

To facilitate the development of a model which allows the dependence of the fretting wear rate upon the scar width to be developed (see Section 4.2), a mathematical relationship between the wear scar width and volume is required; such a relationship is proposed, based upon the assumptions espoused in Figure 9. Since the wear scar width is large compared with the slip amplitude, it is assumed that the wear scar on the flat and cylindrical specimens have the same width and indeed share the same profile. 
It is also assumed that any ploughed material is small and does not result in significant errors in measurement of the wear scar width.

(a)
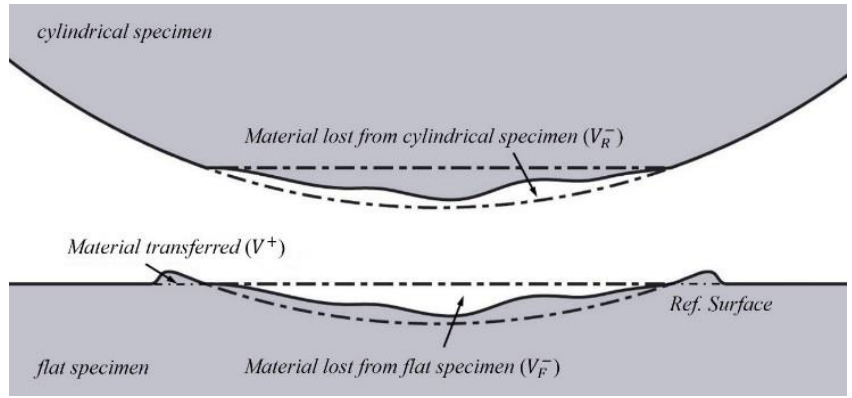

Material lost from flat specimen $\left(V_{F}^{-}\right)$

$\triangle$ Material lost from cylindrical specimen $\left(V_{R}^{-}\right)$

(b)

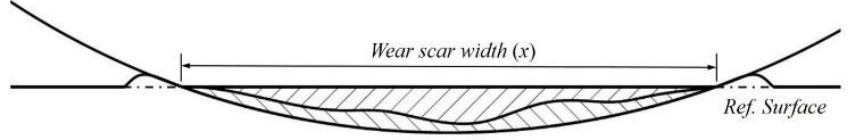

Figure 9: (a) A typical contact of high strength steel after fretting test with a small amount of transferred material at the edge, illustrating the assumption (b) that the combined wear on the two specimens result in a total worn volume equivalent to the minor segment of the cylinder.

With these assumptions, then it can be seen that the total wear volume (i.e. the combined material lost from the cylinder and the flat) is simply the volume of the minor cylindrical segment of intersection between the flat and cylindrical specimens (Figure 9b). Hence, Equation 1 can be simplified as:

$$
V^{W} \approx-V^{-}=-\left(V_{R}^{-}+V_{F}^{-}\right)
$$

Therefore, the relationship between wear scar width $(x)$ and the volume $\left(V^{W}\right)$ can be described as follows:

$$
V^{W}=L\left(R^{2} \arcsin \left(\frac{x}{2 R}\right)-\frac{x}{4} \sqrt{4 R^{2}-x^{2}}\right)
$$

It is noted that in the following derivation, $x \geq 0$. Moreover, in this study, the value of wear scar width, $x$, cannot exceed $10 \mathrm{~mm}$ since this is the width of the cylindrical specimen. Also, as indicated in Figure $3, L$ is $10 \mathrm{~mm}$ in the experimental configuration employed.

Figure 10 shows the measured wear volumes plotted against the measured wear scar widths, with the geometrical relationship indicated by Equation 3. It should be noted that the data in Figure 10 contain additional experimental results for the R- 6 pairs outside of the tests reported in this paper (the additional tests were conducted with the same specimen configuration and material and at the same load and fretting frequency (i.e. $450 \mathrm{~N}$ and $20 \mathrm{~Hz}$ ) but with $\Delta^{*}$ between 10 and $25 \mu \mathrm{m}$ ). The data presented relate to wear scars where the scar is broadly uniform in width across the scar; as can be seen in Figure 6, a uniform scar was formed in the R- 6 pairs following test durations as low as $5 \times 10^{3}$ cycles, whereas for the R-160 pairs, much longer test durations were required $\left(1 \times 10^{5}\right.$ cycles) before a sensible and representative estimate of a scar width could be made. As such, the data for the R-160 pairs do not exist at the lower values of width and volume due to the wear scar not being fully formed in these cases. 
Figure 10 illustrates that for the fully formed wear scars, the measured data fit well with the idealised geometrical relationship, but the fit is better for the R- 6 pairs than for the R-160 pairs. The average difference between the predicted and measured volumes for a given scar width were $14 \pm 8 \%$ and 22 $\pm 7 \%$ for the R-6 and R-160 pairs respectively (with the uncertainty representing one standard deviation). It is notable that the measured wear volume for a given scar width is always less than predicted value, as would be expected given the assumptions made; in addition, it is notable that there is no clear trend in the fractional difference in wear scar width across the range examined.

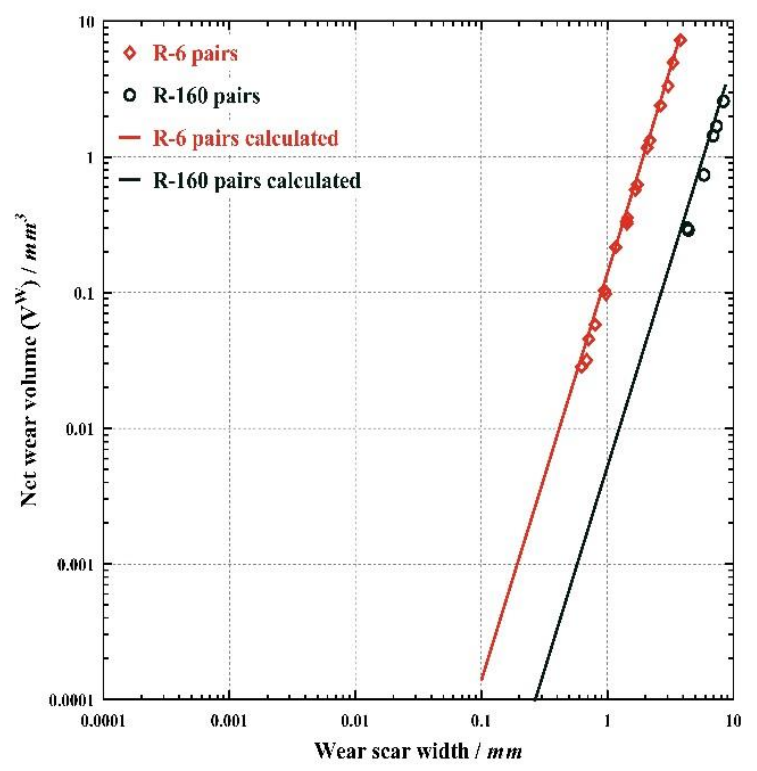

Figure 10: Plot of the wear scar width against net wear volume for fretting tests conducted with R-6 and R-160 pairs at varying displacement amplitude from $\Delta^{*}=10 \mu \mathrm{m}$ to $\Delta^{*}=50 \mu \mathrm{m}$ with $\mathrm{P}=450 \mathrm{~N}$.

\subsection{Modelling the effect of wear scar size on the wear rate}

In the literature addressing the third-body approach in fretting, it is suggested that in certain circumstances, the rate of wear in a fretting contact is limited by the rate of debris egress from the contact (as opposed to the rate of material removal from the opposing surfaces). A schematic diagram suggesting how this may relate to the size of the wear scar was presented earlier in Figure 2. It is proposed that the debris flow will be parallel to the slip direction with a velocity (debris particle displacement per cycle) which is a function of many of the experimental parameters (such as slip amplitude, applied load, temperature, etc.); however, given that none of these were changed during the experiment, it is assumed that any changes in the debris flow during these tests will be associated only with changes in the width of the wear scar as the test proceeds. The physics of the dependence of the debris flow on the wear scar width is not fully understood; however, two proposals are offered, both of which lead to the same dependence of wear rate upon the scar width:

(i) It may be assumed that the debris flow velocity is independent of the wear scar width, which means that the residence time of any debris particle within the contact increases with the wear scar width. If it is assumed that, for wear to proceed, debris must be continually eliminated from the contact, and that the rate of debris elimination controls the rate of wear, then it is proposed that the wear rate will be proportional to the inverse of the debris residence time, i.e. $(1 / x)$.

(ii) Alternatively, it may be assumed that the flow of particles is proportional to the gradient of their concentration between where they are formed (in the contact) and where they are eliminated (the edge of the contact). If it is assumed that their concentrations at the point of formation and elimination are fixed, then the gradient is simply controlled by the 
wear scar width, again leading to the hypothesis that the wear rate will be proportional to $(1 / x)$.

As such, for situations where debris ejection from the contact is the rate determining step (i.e. the region to the right of the vertical line in Figure 2),

$$
\frac{d V^{W}}{d E_{d}}=\frac{k}{x}
$$

where $k$ is a constant which describes the scar width-dependent wear rate of the contacting pair.

So far, relationships between the wear volume and the wear scar width (Equation 3), and the wear scar width and the wear rate (Equation 4) have been determined.

Finding the derivative of $V^{w}$ in terms of $x$ from Equation 3 results in the following equation (see Appendix for details):

$$
\frac{d V^{W}}{d x}=\frac{x^{2} L}{2 \sqrt{4 R^{2}-x^{2}}}
$$

Substituting Equation 5 into Equation 4 gives:

$$
\frac{d E_{d}}{d x}=\frac{x^{3} L}{2 k \sqrt{4 R^{2}-x^{2}}}
$$

which can be integrated to yield the following which describes the relationship between $E_{d}$ and $x$ only when $E_{d} \geq E_{t h}$ (see Appendix for details):

$$
E_{d}=m L\left(16 R^{3}-\sqrt{4 R^{2}-x^{2}}\left(8 R^{2}+x^{2}\right)\right)+E_{t h}
$$

where $m=\frac{1}{6 k}$ and $E_{t h}$ is the energy dissipated when wear first occurs (i.e. when $x$ first becomes a positive number). $E_{t h}$ is often referred to as the threshold energy for onset of wear $[15,22]$.

Although an equation describing the relationship between $E_{d}$ and $V^{W}$ cannot be derived readily, a set of parametric equations based on wear scar width was established. That is, for a known value of $x$, both $E_{d}$ (Equation 7) and $V^{W}$ (Equation 3) can be evaluated. Constants $m$ and $E_{t h}$ for both the R- 6 and R-160 tests were determined from experimental results. To obtain $m$ and $E_{t h}$, it is essential to transform Equation 7 into a linear function by assigning $\left(16 R^{3}-\sqrt{4 R^{2}-x_{i}^{2}}\left(8 R^{2}+x_{i}^{2}\right)\right)$ to $X_{i}\left(R, x_{i}\right), m L$ to $K, E_{t h}$ to $C$ and $E_{d}$ to $Y_{i}\left(R, x_{i}\right)$ (as shown in Equation 8). Therefore, for each of R-6 and R-160 tests, there is a unique set of $\left(X_{i}, Y_{i}\right)$, which is grouped by each radius and then used to solve Equation 8 for the values of $K$ and $C$.

$$
Y_{i}\left(R, x_{i}\right)=K X_{i}\left(R, x_{i}\right)+C \quad i=1 \text { to } n
$$

where $\mathrm{n}$ is number of tests.

Although these two specimen pair geometries (R-6 and R-160) appear to elicit very different wear rates overall (and rates that change with test duration), constants $m$ and $E_{t h}$ evaluated for these two geometries are rather similar. The values of $m$ are as follows (with subscripts indicating the radius of the cylinder in the pair): $m_{6}=2.29 \times 10^{15} \mathrm{~J} \mathrm{~m}^{-4}$ and $m_{160}=2.67 \times 10^{15} \mathrm{~J} \mathrm{~m}^{-4}$, a difference of less than $15 \%$; the corresponding values of $E_{t h}$ for these two sets of data are: $E_{t h \_6}=418 \mathrm{~J}$ and $E_{t h \_160}=702 \mathrm{~J}$ 
(a difference of less than 50\%). To demonstrate that this methodology is appropriate and that it robustly describes these two sets of data and their evolution with test duration, a fit was produced for each dataset using common values of $m$ and $E_{t h}$; specifically, the mean values of $m$ and $E_{t h}$ were employed, namely $m=2.48 \times 10^{15} \mathrm{~J} \mathrm{~m}^{-4}$ and $E_{t h}=560 \mathrm{~J}$. Figure 11 shows the experimental data plotted against the calculated values for both R-6 and R-160 pairs; the validity of the methodology and hypothesis is clear from the correlation between the data and the predictions.

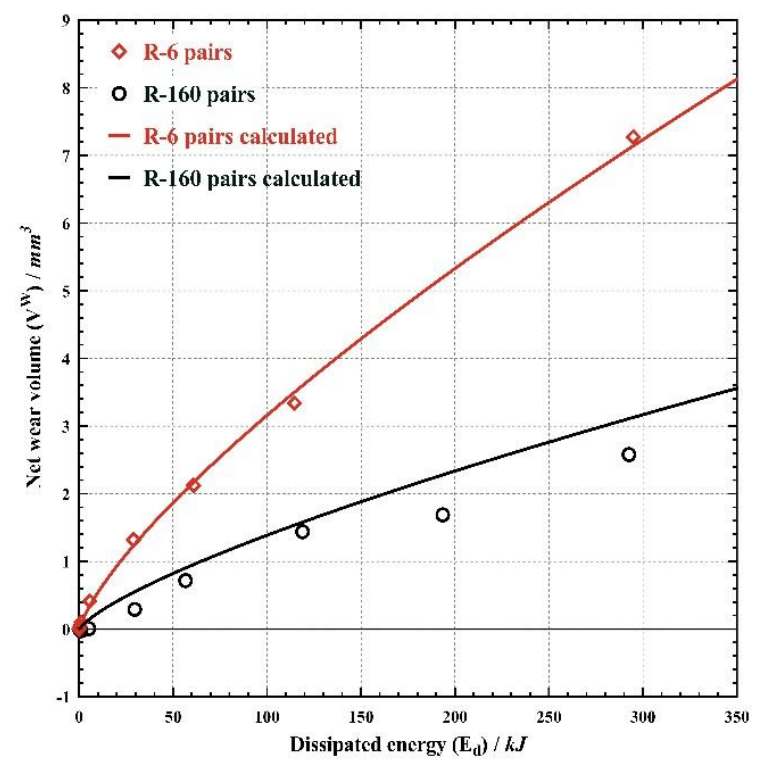

Figure 11: A comparison between the experimental data and the calculated values based on Equation 3 and Equation 7 showing the wear volume as a function of dissipated energy for fretting tests conducted with R-6 pairs and $R-160$ pairs.

\section{Discussion}

\subsection{Wear scar characteristics}

In previous research that has examined the effects of specimen geometry on the fretting wear behaviour of non-conforming contact pairs $[5,13,14,17]$, it can been seen that more conforming contacts result in lower wear rates (as has been seen in this work). In the study conducted by Warmuth et al. which used the same steel as in this work $[13,14]$, it was observed that R- 6 geometries resulted in U-shaped wear scars, whereas R-160 geometries resulted in large pits and peaks on the wear surfaces which were associated with metallic transfer between the specimens. However, in these papers, the fretting tests were conducted only for a small number of cycles $\left(1 \times 10^{5}\right.$ cycles). It was proposed by Warmuth et al. [13] that these pit-peak features were formed when the rate of oxygen ingress into the contact was too low to result in oxidation of the nascent metal surfaces formed as part of the wear process before metal-metal transfer could occur, and that more conforming contacts restricted this oxygen ingress due to the larger widths of the fretting contacts

In the current work, it can be seen that these pit-peak features were never observed for tests with the R-6 geometry (additional tests with numbers of cycles as low as 500 were conducted, but U-shaped scars were always observed), and it is proposed that this is due to the ease of oxygen ingress into these contacts. However, whilst the pit-peak features were observed for tests with small numbers of cycles $\left(<1 \times 10^{5}\right.$ cycles) with the R-160 pairs, they were eliminated at higher numbers of cycles $\left(>1 \times 10^{6}\right.$ cycles), despite the fact that the scar widths were getting larger as the wear proceeded (which presumably further restricts oxygen ingress into the scar). It is proposed that whilst oxygen ingress into the scar will become more limited by increases in the wear scar width associated with wear, the corresponding increase in the restriction of wear debris expulsion from the contact (and the associated 
reduction in wear rate) is the dominant effect; hence, as the scars grow at higher numbers of cycles, the oxygen ingress ceases to be a rate-determining (and, therefore, a mechanism-determining) process, with the debris expulsion from the scar taking this role.

These observations also indicate the need to examine the mechanisms of fretting wear across a wide range of durations; it was proposed in earlier work [13] that the pit-peak features may be significant in terms of the tendency for fretting fatigue. However, for many components in service, the numbers of fretting cycles are much larger than those typically employed in laboratory tests; given the changes in mechanism observed here, it is argued that misleading conclusions may be drawn if the test conditions do not replicate those seen in service or can be demonstrated to have reached a steadystate.

\subsection{Wear rate dependence on contact width in fretting}

Whilst the general concept of the competition between debris-formation and debris-expulsion to control the wear mechanisms and rates of wear has been well accepted since the publications of Godet and co-workers [7], a physically-based quantitative model is initially outlined in this study which proposes that in situations where the rate of wear in fretting is limited by debris expulsion from the contact, the wear rate is inversely proportional to the width of the scar. The model is dependent upon the observation that the total wear volume of the fretting pair is very close to that of a minor segment of the cylinder defined by the wear scar width. The model is in good agreement with the experimental data and is able to predict the evolution of wear volumes with fretting duration for two very different contact geometries in which the wear volumes are clearly seen to be very dependent upon these geometrical differences.

It is not clear where the transition from the debris-formation controlled regime and the debrisexpulsion controlled regime occurs (see Figure 2), but given the correlation between the experimental data and the predictions based upon the hypothesis, it may be assumed that this transition occurs at relatively small wear scar widths (i.e. the wear rate is debris-expulsion controlled from an early stage). The exceedingly high rates of wear that the model predicts as the scar width tends towards zero are not observed for two reasons; (i) the minimum scar widths (at the beginning of the test) are finite due to elastic deformation; the initial Hertzian contact widths (full width) under the test conditions are 111 $\mu \mathrm{m}$ and $572 \mu \mathrm{m}$ for the R- 6 and R-160 pairs respectively; (ii) at small scar widths, debris expulsion ceases to be the rate-determining mechanism, with the rate of debris formation itself taking that role instead.

\subsection{Consideration of debris expulsion effects in research programmes}

As discussed, the role of debris expulsion from a fretting contact is more significant than it is in sliding wear in controlling both the rates and mechanisms of wear. However, much fretting research (particularly that which seeks to develop new fretting resistant materials or compares the behaviour of different materials) simply considers the wear rates without considering whether the effects observed are associated with changes in the rate of debris formation or with changes in the rate of debris ejection from the contact.

Although this paper has focussed on the influence of the wear scar size (both initial size and evolved size in a non-conforming contact due to the wear process itself) on the rates of wear (controlled by debris expulsion), it is recognised that many other factors also control debris expulsion from the scar, including initial geometry (e.g. flat-on-flat, sphere-on-flat) and contact size, fretting stroke, fretting frequency, temperature, hardness of the contacting materials etc [16, 23-26]. As such, it is argued that consideration of debris expulsion from a contact (in particular, via SEM analysis of the wear scars) is a critical part of research in the field of fretting, and that caution needs to be exercised when comparing 
wear rates in situations in which an analysis of the debris expulsion from the contact has not been considered.

\section{Conclusions}

In fretting wear, the wear rate is either controlled by the rate of debris formation within the contact or by the rate of debris ejection from the contact. In this work, it was observed that, in fretting wear of contacts with a cylinder on flat geometry, the wear rate is controlled by the rate of debris ejection form the contact, which is dependent upon the evolving size of the wearing contact. The instantaneous wear rate has been shown to be inversely proportional to the wear scar width, meaning that changes in wear rate with initial contact geometry and the duration of fretting can be quantitatively explained. The validity of the model is demonstrated by its ability to account for significant differences in measured wear rate (with either geometry of test duration). The model thus indicates that fretting wear is contact size-dependent and cannot be described adequately by an Archard-type formulation. It is noted that this conclusion relates only to a cylinder-on-flat configuration, and that for other contact geometries (e.g. annular ring contact or ball-on-flat contact), the dependence of the rate of debris expulsion from the contact on the contact size will be different.

For the geometry employed in this work, the development of the relationship between wear scar width and wear rate required a relationship between the wear scar volume and width. A simple geometrical relationship was proposed and validated by the agreement between the measured data and predicted values.

Finally, it is noted that for the R-160 contact pairs, the wear mechanism changed with the test duration. At low cycles (up to $1 \times 10^{5}$ cycles), the rate of oxygen ingress to the wearing surfaces in the contact was not high enough to facilitate the development of an oxide debris bed between the first bodies, and this resulted in pit-peak features on the contact surfaces which were associated with metallic transfer between the specimens. As the test duration was extended (above up to $1 \times 10^{6}$ cycles), the rate at which debris was expelled from the contact reduced the overall wear rate sufficiently, so that the rate of oxygen ingress was high enough to facilitate the formation of an oxide debris bed, and a Ushaped wear scar was formed (as was always observed for the less conforming R-6 pairs). This observation indicates that care must be taken in fretting testing to ensure that the duration is long enough to ensure that steady-state wear mechanisms are operative.

Competing interests We have no competing interests.

Funding statement $\mathrm{TZ}$ is a PhD student at the University of Nottingham and his work and studentship are funded by the University of Nottingham.

Acknowledgements The authors wish to thank the University of Nottingham for both financial and facility support. 


\section{References}

1. ASTM, Standard Terminology Relating to Wear and Erosion. ASTM International, 2015. ASTM G40-15.

2. Brown, S., Ed., Material Evaluation Under Fretting Conditions. ASTM International, 1982. STP780-EB.

3. Meng, H.C. and K.C. Ludema, Wear models and predictive equations: their form and content. Wear, 1995. 181 - 183: p. 443 - 457.

4. $\quad$ Archard, J.F., Contact and Rubbing of Flat Surfaces. Journal of Applied Physics, 1953. 24(8): p. 981 - 988.

5. Fouvry, S., C. Poulins, and S. Deyber, Impact of contact size and complex gross-partial slip conditions on Ti-6Al-4V/ Ti-6Al-4V fretting wear. Tribology International, 2009. 42(3): p. 460 - 473.

6. Fouvry, S., et al., An energy description of wear mechanisms and its applicatons to oscillation sliding contacts. Wear, 2003. 255(1 - 6): p. 287 - 298.

7. Godet, M., The third-body approach: a mechanical view of wear. Wear, 1984. 100(1 - 3): p. 437 - 452.

8. Fillot, N., I. lordanoff, and Y. Berthier, Wear modeling and the third body concept. Wear, 2007. 262(7 8): p. 949 - 957.

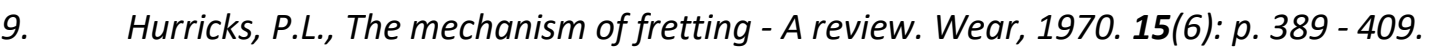

10. Godet, M., Third-bodies in tribology. Wear, 1990. 136(1): p. 29 - 44.

11. Berthier, Y., Experimental evidence for friction and wear modeling. Wear, 1990. 139(1): p. 77 - 92.

12. Waterhouse, R.B., Fretting Corrosion. International Series of Monographs on Material Science and Technology, ed. D.W. Hopkins. Vol. 10. 1972: Pergamon Press. 88.

13. Warmuth, A.R., P.H. Shipway, and W. Sun, Fretting wear mapping: The influence of contact geometry and frequency on debris formation and ejection for a steel-on-steel pair. Proceedings of the Royal Society A: Mathematical, Physical and Engineering Science, 2015. 471(2178).

14. Warmuth, A.R., et al., The effect of contact geometry on fretting wear rates and mechanism for a high strength steel. Wear, 2013. 301(1 - 2): p. 491 - 500.

15. Pearson, S.R. and P.H. Shipway, Is the wear coefficient dependent upon slip amplitude in fretting? Vingsbo and Söderberg revisited. Wear, 2015. 330 - 331(May - June 2015): p. 93 - 102.

16. Hayes, E.K. and P.H. Shipway, Effect of test conditions on the temperature at which a protective debris bed is formed in fretting of a high strength steel. Wear, 2017. 376 - 377(Part B): p. 1460 - 1466.

17. Merhej, $R$. and S. Fouvry, Contact size effect on fretting wear behaviour: application to an AISI 52100/AISI 52100 interface. Lubrication Science, 2009. 21: p. 83-102.

18. Berthier, Y., L. Vincent, and M. Godet, Velocity accommodation in fretting. Wear, 1988. 125(1 - 2): $p$. $25-28$.

19. Mohd Tobi, A.L., et al., The effect of gross sliding fretting wear on stress distributions in thin W-DLC coating systems. Tribology International, 2010. 43(10): $p$. 1917-1932.

20. Leen, S.B., et al., Macroscopic fretting variables in a splined coupling under combined torque and axial load. Journal of Strain Analysis for Engineering Design, 2001. 36: p. 481 - 497.

21. Elleuch, K. and S. Fouvry, Wear analysis of A357 aluminium alloy under fretting. Wear, 2002. 253(5): $p$. 662-672.

22. Sauger, E., et al., Tribologically transformed structure in fretting. Wear, 2000. 245(1-2): p. 39-52.

23. Jin, X., P.H. Shipway, and W. Sun, The role of frictional power dissipation (as a function of frequency) and test temperature on contact temperature and the subsequent wear behaviour in a stainless steel contact in fretting. Wear, 2015. 330-331: p. 103-111.

24. Jin, X., W. Sun, and P.H. Shipway, The role of geometry changes and debris formation associated with wear on the temperature field in fretting contacts. Tribology International, 2016. 102: $p$. 392-406.

25. Lemm, J.D., et al., The influence of surface hardness on the fretting wear of steel pairs-Its role in debris retention in the contact. Tribology International, 2014. 81: p. 258-266.

26. Pearson, S.R., et al., The effect of temperature on wear and friction of a high strength steel in fretting. Wear, 2013. 303(1-2): p. 622-631. 


\section{Appendix}

The volume of a minor segment of a cylinder (parallel to the cylinder axis) is given by the following equation:

$$
V^{W}=L\left(R^{2} \arcsin \left(\frac{x}{2 R}\right)-\frac{x}{4} \sqrt{4 R^{2}-x^{2}}\right)
$$

where terms are as previously defined. In this study, it is proposed that the wear rate $\frac{d V^{W}}{d E_{d}}$ is dependent upon the scar width $(x)$ as follows:

$$
\frac{d V^{W}}{d E_{d}}=\frac{k}{x}
$$

which can be rewritten as follows:

$$
\frac{d V^{W}}{d x} \frac{d x}{d E_{d}}=\frac{k}{x}
$$

The two individual terms can be defined as follows.

To find $\frac{d V^{W}}{d x}$, Equation $A 1$ can be differentiated as follows:

$$
\frac{d V^{W}}{d x}=L R^{2} \frac{d}{d x}\left(\arcsin \left(\frac{x}{2 R}\right)\right)-\frac{L}{4} \frac{d}{d x}\left(x \sqrt{4 R^{2}-x^{2}}\right)
$$

Defining:

$$
\begin{aligned}
& h(x)=\arcsin \left(\frac{x}{2 R}\right) \\
& j(x)=x \sqrt{4 R^{2}-x^{2}}
\end{aligned}
$$

this can be rewritten as follows:

$$
\frac{d V^{W}}{d x}=L R^{2} \frac{d}{d x}(h(x))-\frac{L}{4} \frac{d}{d x}(j(x))
$$

Finding the derivative of $h(x)$ with respect to $x$ yields:

$$
\frac{d}{d x}(h(x))=\frac{1}{2 R} \frac{1}{\sqrt{1-\left(\frac{x}{2 R}\right)^{2}}}=\frac{1}{\sqrt{4 R^{2}-x^{2}}}
$$

Finding the derivative of $j(x)$ with respect to $x$ yields:

$$
\frac{d}{d x}(j(x))=\sqrt{4 R^{2}-x^{2}}-x \frac{1}{2} \frac{2 x}{\sqrt{4 R^{2}-x^{2}}}=\frac{4 R^{2}-2 x^{2}}{\sqrt{4 R^{2}-x^{2}}}
$$


Therefore, by substituting the expressions of $\frac{d}{d x}(h(x))$ and $\frac{d}{d x}(j(x))$ into $\frac{d V^{W}}{d x}$ gives:

$$
\frac{d V^{W}}{d x}=L R^{2} \frac{1}{\sqrt{4 R^{2}-x^{2}}}-\frac{L}{4} \frac{4 R^{2}-2 x^{2}}{\sqrt{4 R^{2}-x^{2}}}=\frac{x^{2} L}{2 \sqrt{4 R^{2}-x^{2}}}
$$

Substituting Equation $\mathrm{A} 3$ into Equation $\mathrm{A} 2$ gives:

$$
d E_{d}=\frac{x^{3} L}{2 k \sqrt{4 R^{2}-x^{2}}} d x
$$

which can be integrated as follows:

$$
E_{d}=\frac{L}{2 k} \int \frac{x^{3}}{\sqrt{4 R^{2}-x^{2}}} d x
$$

Defining that:

$$
k(x)=\frac{x^{3}}{\sqrt{4 R^{2}-x^{2}}}
$$

and

$$
u=x^{2}
$$

then

$$
E_{d}=\frac{L}{2 k} \int k(x) d x
$$

and

$$
d u=2 x d x
$$

This returns:

$$
\int k(x) d x=\int \frac{u x}{\sqrt{4 R^{2}-u}} \frac{d u}{2 x}=\frac{1}{2} \int \frac{u}{\sqrt{4 R^{2}-u}} d u
$$

Defining:

$$
v=4 R^{2}-u
$$

then

$$
d v=-d u
$$

Combing $u(x), v(u)$ and their corresponding differentiation into $\int k(x)$ and rearranging the equation yields:

$$
\int k(x) d x=\frac{-1}{2} \int \frac{4 R^{2}-v}{\sqrt{v}} d v=\frac{1}{2} \int \sqrt{v} d v-2 R^{2} \int \frac{1}{\sqrt{v}} d v
$$

and thus 


$$
\int k(x) d x=\frac{1}{3} v^{\frac{3}{2}}-4 r^{2} v^{\frac{1}{2}}+c_{1}
$$

By substituting the expressions for $v(u)$ and $u(x)$ :

$$
\begin{gathered}
\int k(x) d x=\frac{1}{3}\left(4 R^{2}-u\right)^{\frac{3}{2}}-4 R^{2}\left(4 R^{2}-u\right)^{\frac{1}{2}}+c_{1}=\frac{1}{3}\left(4 R^{2}-x^{2}\right)^{\frac{3}{2}}-4 R^{2}\left(4 R^{2}-x^{2}\right)^{\frac{1}{2}}+c_{1} \\
\int k(x) d x=\sqrt{4 R^{2}-x^{2}}\left(\frac{1}{3}\left(4 R^{2}-x^{2}\right)-4 R^{2}\right)+c_{1}=-\frac{1}{3} \sqrt{4 R^{2}-x^{2}}\left(8 R^{2}+x^{2}\right)+c_{1}
\end{gathered}
$$

Therefore:

$$
E_{d}=\frac{L}{2 k}\left(-\frac{1}{3} \sqrt{4 R^{2}-x^{2}}\left(8 R^{2}+x^{2}\right)\right)+\frac{L}{2 k} c_{1}
$$

Simplifying the terms here yields:

$$
E_{d}=-m L\left(\sqrt{4 R^{2}-x^{2}}\left(8 R^{2}+x^{2}\right)\right)+C
$$

where: $C=\frac{L}{2 k} c_{1}$ and $m=\frac{1}{6 k}$

To evaluate the integration constant, $C$, it is noted that in a fretting contact, there is a threshold of energy dissipated, $E_{t h}$, below which $x=0$ (i.e. there is no wear) $[15,22]$; in this region, Equation A5 does not describe the relationship between $E_{d}$ and $x$. However, once $E_{d}$ has exceeded $E_{t h}$, then wear occurs (and thus $x>0$ ). Evaluating Equation $\mathrm{A} 5$ when $E_{d}=E_{t h}$ and $x=0$ yields the following:

$$
E_{t h}=-m L\left(16 R^{3}\right)+C
$$

and thus

$$
C=E_{t h}+16 m L R^{3}
$$

The final equation for $E_{d}$ is therefore as follows:

$$
E_{d}=m L\left(16 R^{3}-\sqrt{4 R^{2}-x^{2}}\left(8 R^{2}+x^{2}\right)\right)+E_{t h}
$$

